PART I

GAMMA-RAY ASTRONOMY 


\title{
THE PRESENT STATE OF GAMMA-RAY ASTRONOMY*
}

\author{
G. CLARK \\ Dept. of Physics and Center for Space Research, Massachusetts Institute of Technology, \\ Cambridge, Mass., U.S.A.
}

\begin{abstract}
The current state of gamma-ray astronomy is reviewed in terms of the most recent experimental results that define, in either measured fluxes or upper limits, the cosmic photon spectrum in the energy range from $0.5 \mathrm{MeV}$ to $10^{16} \mathrm{eV}$. Methods of research developed during the last $10 \mathrm{yr}$ are discussed and specific results are cited. Cosmic gamma rays have been observed in two regions of the spectrum - from 0.5 to $6 \mathrm{MeV}$ and from about $50 \mathrm{MeV}$ to several hundred $\mathrm{MeV}$. Above $50 \mathrm{MeV}$ a strong concentration of intensity is observed in a band of directions around the galactic equator. In both spectral regions a diffuse background gamma ray flux, apparently isotropic and of extragalactic origin, is observed. Evidence of $\sim 50 \mathrm{MeV}$ gamma-ray pulses from the Crab pulsar has been reported. No definite evidence of any other discrete gamma-ray source besides the galaxy itself has been observed at any energy.
\end{abstract}

By 1952 the processes which now appear to be the most important for the production of cosmic gamma rays had been described in three fundamental papers. Feenberg and Primakoff (1948) had calculated the degradation of high energy cosmic electrons by the inverse Compton scattering of starlight which produces high energy recoil photons. Hutchinson (1952) had discussed the production of bremsstrahlung gamma rays by cosmic electrons in collisions with matter. Hayakawa (1952) had pointed out that collisions of cosmic ray protons with interstellar matter produce $\pi^{\circ}$ mesons which decay into gamma rays. These and many subsequent theoretical discussions offered the prospect of clear and calculable relations between gamma-ray fluxes and several of the most important parameters of celestial objects such as magnetic field strengths, matter densities and radiation intensities. This, in turn, has stimulated many efforts over the past twenty years to detect and measure cosmic gamma rays over the spectral range from 0.5 to $10^{16} \mathrm{eV}$.

The earliest exploratory observations, however, predated most of the relevant theoretical work. Hulsizer and Rossi (1949) set an upper limit of several percent on the relative intensity of primary electromagnetic radiation above $1 \mathrm{GeV}$ compared to cosmic rays in a balloon experiment with an ion chamber. Critchfield et al. (1952) reduced this limit to $0.6 \%$ in a balloon cloud chamber experiment. Perlow and Kissinger (1951) placed an upper limit of about $10^{-2}\left(\mathrm{~cm}^{2} \mathrm{~s} \text { ster }\right)^{-1}$ on the flux of 3 to $90 \mathrm{MeV}$ gamma rays with Geiger tube telescopes in a series of V-2 rocket flights.

Although a number of experimental searches were carried out during the next ten years, it was not until the decade of the 1960's that the first positive observations were made. Gamma rays with energies around $1 \mathrm{MeV}$ and apparently of extragalactic origin were detected in an experiment on the Ranger III Moon probe (Arnold et al., 1962), and galactic and extragalactic gamma rays with energies near $100 \mathrm{MeV}$ were detected by an experiment on the OSO-3 satellite (Clark et al., 1968).

* Supported in part by the National Aeronautics and Space Administration under contract NGL22-009-372. 
The purpose of this review is to describe the current state of gamma-ray astronomy in terms of the most recent experimental results that define, in either measured fluxes or upper limits, the cosmic photon spectrum in the energy range from $0.5 \mathrm{MeV}$ to $10^{16} \mathrm{eV}$. A more detailed review of recent theoretical and experimental developments has been published by Fazio (1970).

It is convenient to divide the gamma-ray spectrum into several parts corresponding to the effective energy ranges of the principal experimental methods. This division, however, is essentially arbitrary from an astrophysical point of view since several of the processes which produce cosmic gamma rays may well be significant over the entire ten decades of the spectrum. Moreover, there are gaps between the effective energy ranges of the available experimental methods.

\section{0.5 to $10 \mathrm{MeV}$}

Astronomical observations in the low energy range from 0.5 to $10 \mathrm{MeV}$ are plagued by two severe problems. The first is interference from secondary gamma rays generated by charged cosmic rays in material that subtends a substantial solid angle at the detector, e.g., the shielding around the detector, the vehicle in which the detector is carried, or the atmosphere, as in the case of a balloon vehicle or a satellite in nearEarth orbit. The second problem is the difficulty of achieving directional sensitivity. The interaction products of a low energy gamma ray retain little information about the incident direction. As a result, methods of coincidence detection and track imaging are ineffective. At the same time, gamma rays above $0.5 \mathrm{MeV}$ have relatively long mean-free-paths so that geometrical collimation requires massive shielding which is itself a source of background. This background can be suppressed by the use of 'active' shielding, i.e., shielding composed of a high- $Z$ scintillator in anticoincidence with the detector. This approach has been used successfully in the study of high energy cosmic X-ray sources up to several hundred $\mathrm{keV}$. At higher energies, however, the residual unsuppressed background generated within the shielding and associated equipment again dominates the signal produced by cosmic gamma rays entering the open aperture.

In the ERS-18 experiment (Vette et al., 1970), as in the earlier one on the Ranger 3, no direct attempt was made to achieve directional sensitivity. The detector was a $3^{\prime \prime} \times 3^{\prime \prime} \mathrm{NaI}(\mathrm{Na})$ scintillator with a thin anticoincidence jacket that eliminated events directly produced by incident charged particles. The measurements were made far enough from Earth so that the flux of Earth-albedo gamma rays was negligible. The entire payload, including the satellite payload itself, was made so light $(7.8 \mathrm{~kg})$ that the counting rate due to the locally produced gamma rays was demonstrably small compared to the measured rate of events. Since the signal was far above 'noise', a clear comparison could be made between the observed pulse size distribution and calculated distributions derived by convolving hypothetical primary energy spectra with the known response function of the detector.

Below $1 \mathrm{MeV}$ the ERS-18 results confirm the Ranger-3 results. However, the striking 
conclusion from the ERS-18 is that the observed pulse size distribution in the range corresponding to primary energies above $1 \mathrm{MeV}$ is not consistent with the hypotheses that the cosmic gamma-ray spectrum is a simple power law fitted to the previously measured diffuse cosmic photon spectrum over the range $50-220 \mathrm{keV}$ or that it is the more complicated spectrum derived by Brecher and Morrison (1968) for the intergalactic Compton recoil photon flux (see Figure 1). In either case, the observed size distribution shows a marked excess of counts in the 1 to $6 \mathrm{MeV}$ range which implies the existence of an anomaly which raises the flux density at $3 \mathrm{MeV}$ above the $E^{-2.3}$ spectrum by about a factor of 5 .

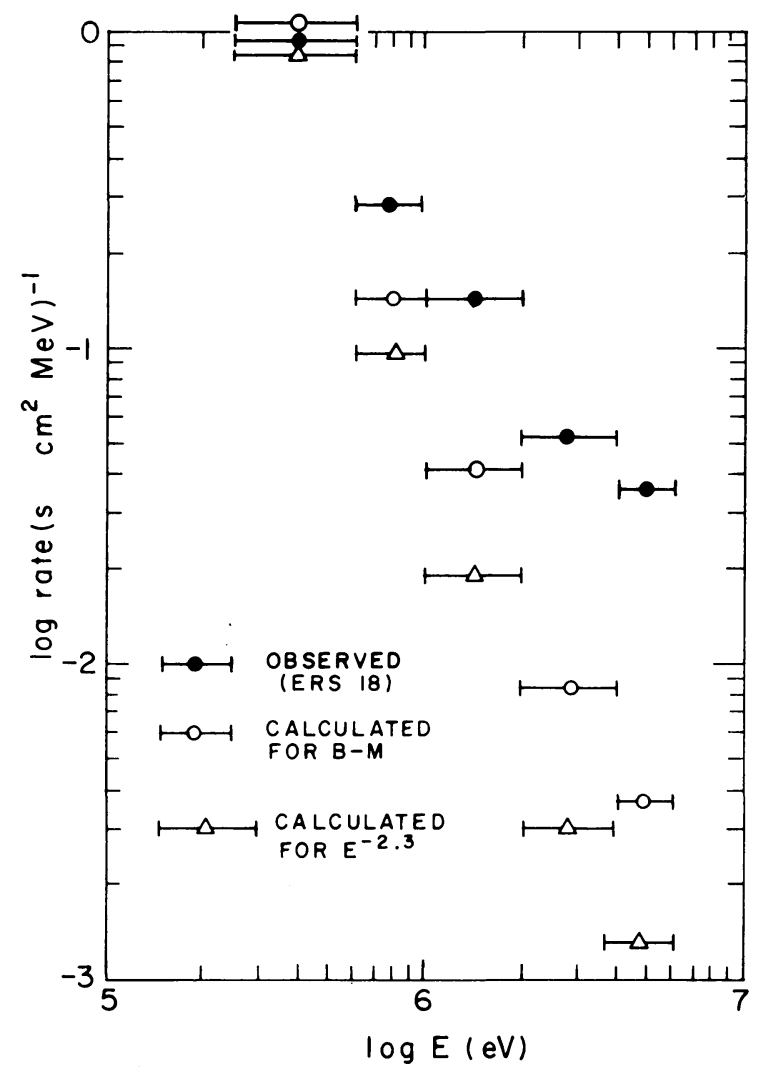

Fig. 1. Comparison between the counting rates observed in the ERS-18 experiment and the rates predicted for a cosmic spectrum conforming to (a) an $E^{-2.3}$ power law, and (b) the Brecher-Morrison spectrum for inverse Compton scattered photons.

In the absence of direct information about the directions of the observed gamma rays, Vette et al. show that their rate is too high to be plausibly accounted for in terms of sources in the galactic disk. Their argument rests on the observed properties of galactic emission at lower (X-ray) and higher $(\sim 100 \mathrm{MeV})$ energies. They conclude that the observed flux is most probably isotropic and of extragalactic origin. 
Clayton and Silk (1969) have suggested that a 1-3 MeV anomaly may be caused by nuclear gamma rays from the ${ }^{56} \mathrm{Ni}$ decay chain emitted by the ejecta of distant supernovas. They predict a spectrum smeared by the red shift towards lower energies from the line of ${ }^{56} \mathrm{Co}$ at $3.47 \mathrm{MeV}$. This hypothesis, however, appears to fall short of a qualitative explanation of the observed anomaly which extends to $6 \mathrm{MeV}$.

Stecker (1969) has attributed the anomaly to gamma rays that arose from the decay of $\pi^{\circ}$ mesons produced in an early epoch of higher cosmic ray intensity. The spectrum of such gamma rays at production has a peak at $m_{\pi^{\circ}} c^{2} / 2 \sim 70 \mathrm{MeV}$. Stecker suggests that it is this spectral feature, transformed by the red shift from $Z \sim 100$, that now appears as the 1-6 MeV anomaly.

\section{2. $30 \mathrm{MeV}$ to $1 \mathrm{GeV}$}

Above a few $\mathrm{MeV}$ the most probable interaction between a gamma ray and matter is pair conversion from which an electron-positron pair emerges with a total momentum close to that of the incident photon. Directional observations in this energy range can be made, in principle, with coincidence counter telescopes and spark chambers which measure the direction and energy of these pairs. However, from the pair-conversion threshold energy near $1.0 \mathrm{MeV}$ to several tens of $\mathrm{MeV}$ practical difficulties are caused by the wide angle between the trajectories of the two electrons, by the large scattering of the electrons, both at the site of production and in the material through which they pass, and by the short range of the electrons. As a result, the positive observational results which have been published so far are confined to the range between an energy threshold near $50 \mathrm{MeV}$ and an intensity threshold of several hundreds of $\mathrm{MeV}$.

The first definite observations of cosmic gamma rays in the energy range above $50 \mathrm{MeV}$ were made with a coincidence counter telescope on the OSO-3 satellite (Clark et al., 1968). The instrument had a pair converter in the form of a multi-layer scintillator, a directional Cerenkov counter, an energy detector in the form of a layered assembly of tungsten and scintillator slabs, and an anticoincidence shield to reject events produced by, or associated with, charged particles that strike the shield.

The energy response of the OSO-3 instrument has a threshold near $70 \mathrm{MeV}$ and rises to a plateau above $300 \mathrm{MeV}$. The angular response is represented approximately by a Gaussian of the form $\exp \left[-\left(\theta / \theta_{0}\right)^{2}\right]$ with $\theta_{0}=15^{\circ}$. The performance in orbit showed no substantial deterioration in sixteen months during which time the instrument recorded over 600 gamma-ray events from directions well above the Earth's horizons.

The absolute fluxes that were derived from the OSO-3 results on the basis of the detector efficiency calculated from pre-launch calibration data were surprisingly high in the light of theoretical expectations for galactic gamma-ray production. Moreover, discrepancies were found between the intensities of Earth albedo gamma rays obtained from the OSO-3 experiment, the earlier results from Explorer 11 and the recent results derived from balloon spark chamber data by Fichtel and Kniffen (1968). In order to reduce the uncertainty in the derived fluxes, additional calibration measurements were made on the back-up instrument of the OSO-3 experiment. These resulted in the dis- 
covery of an error in the previous calibration procedure and a downward revision by a factor near 3 in the absolute fluxes derived from the observed counting rates.

Figure 2 is a gamma-ray sky map in celestial coordinates. The observed rate of gamma-ray events from each $15^{\circ} \times 15^{\circ}$ element is indicated by the number of vertical lines. One can readily perceive a band of higher intensity around the galactic equator, and a maximum towards the galactic center in Sagittarius. The average rate of events recorded when the detector axis was in the galactic latitude interval $-15^{\circ} \leqslant b^{\mathrm{II}} \leqslant 15^{\circ}$ exceeded the overall sky average by $10 \sigma$, and the average over the region $\left|b^{\mathrm{II}}\right| \geqslant 15^{\circ}$, by $19 \sigma$. Thus the evidence for anisotropy correlated with galactic structure is conclusive.

No statistically significant local concentrations of events outside the galactic band are seen. However, because of the low angular resolution of the OSO-3 instrument, this negative result does not place as low upper limits on the intensities of potential discrete sources as do the results derived from the high resolution surveys that have been carried out with balloon-borne spark chambers.

The events recorded from high galactic latitude show no systematic variation in rate with geomagnetic latitude as the satellite traverses its orbit. This is good evidence that most of them are not spurious events caused by ordinary cosmic rays, the intensity of which varies by more than a factor of 2 over the orbit. If one assumes that they are produced by an isotropic component of rays, their rate implies that the intensity for $E_{\gamma}>100 \mathrm{MeV}$ averaged over galactic latitudes $\left|b^{1 \mathrm{II}}\right| \geqslant 30^{\circ}$ is $3 \times 10^{-5}\left(\mathrm{~cm}^{2} \mathrm{~s} \mathrm{sr}\right)^{-1}$. After substracting the events attributable to the isotropic component, the average equivalent line intensity of gamma rays for $E_{\gamma}>100 \mathrm{MeV}$ around the galactic equator in the longitude interval $40^{\circ}<l^{\mathrm{Il}}<340^{\circ}$ is found to be $5 \times 10^{-5}\left(\mathrm{~cm}^{2} \mathrm{~s} \mathrm{rad}\right)^{-1}$. In the interval $330^{\circ}<l^{\mathrm{II}}<30^{\circ}$, which includes the center, the average is $11 \times 10^{-5}\left(\mathrm{~cm}^{2} \mathrm{~s} \mathrm{rad}\right)^{-1}$.

The OSO-3 results have been supported by several more recent observations with other instruments. Kniffen and Fichtel (1970) observed the galactic center region with a $0.25 \mathrm{~m}^{2}$ spark chamber during a six hour flight at an atmospheric depth of $2.9 \mathrm{~g} \mathrm{~cm}^{-2}$. They found a $4.3 \sigma$ excess of counts above background from the galactic plane in the direction of the galactic center, and from this data they compute an equivalent line intensity for $E_{\gamma}>100 \mathrm{MeV}$ of $(2.0 \pm 0.7) \times 10^{-4}\left(\mathrm{~cm}^{2} \mathrm{~s} \mathrm{rad}\right)^{-1}$. Other positive results of lesser statistical significance have been reported by Sood (1969), Valdez and Waddington (1969a) and Hutchinson et al. (1970).

On the other hand, Frye et al. (1969) observed the galactic center region with a balloon-borne spark chamber and report a negative result form which they deduce an upper limit of $7 \times 10^{-5}\left(\mathrm{~cm}^{2} \mathrm{~s} \mathrm{rad}\right)^{-1}$ on the equivalent line intensity for $E_{\gamma}>50 \mathrm{MeV}$. Considering that the spectrum is undoubtedly decreasing from 50 to $100 \mathrm{MeV}$, the contradiction between the results of Frye et al. and those of OSO-3 and KniffenFichtel is a large one. In addition, Frye et al. report a localized $\sim 3 \sigma$ peak which they attribute to a discrete gamma-ray source in Sagittarius with an intensity of $(3 \pm 1) \times 10^{-5}\left(\mathrm{~cm}^{2} \mathrm{~s} \mathrm{rad}\right)^{-1}$ above $50 \mathrm{MeV}$. Although this is not in disagreement with the low angular resolution results from the OSO-3, it is contradicted by the spark chamber data of Kniffen and Fichtel (1970) which place an upper limit at the $95 \%$ 


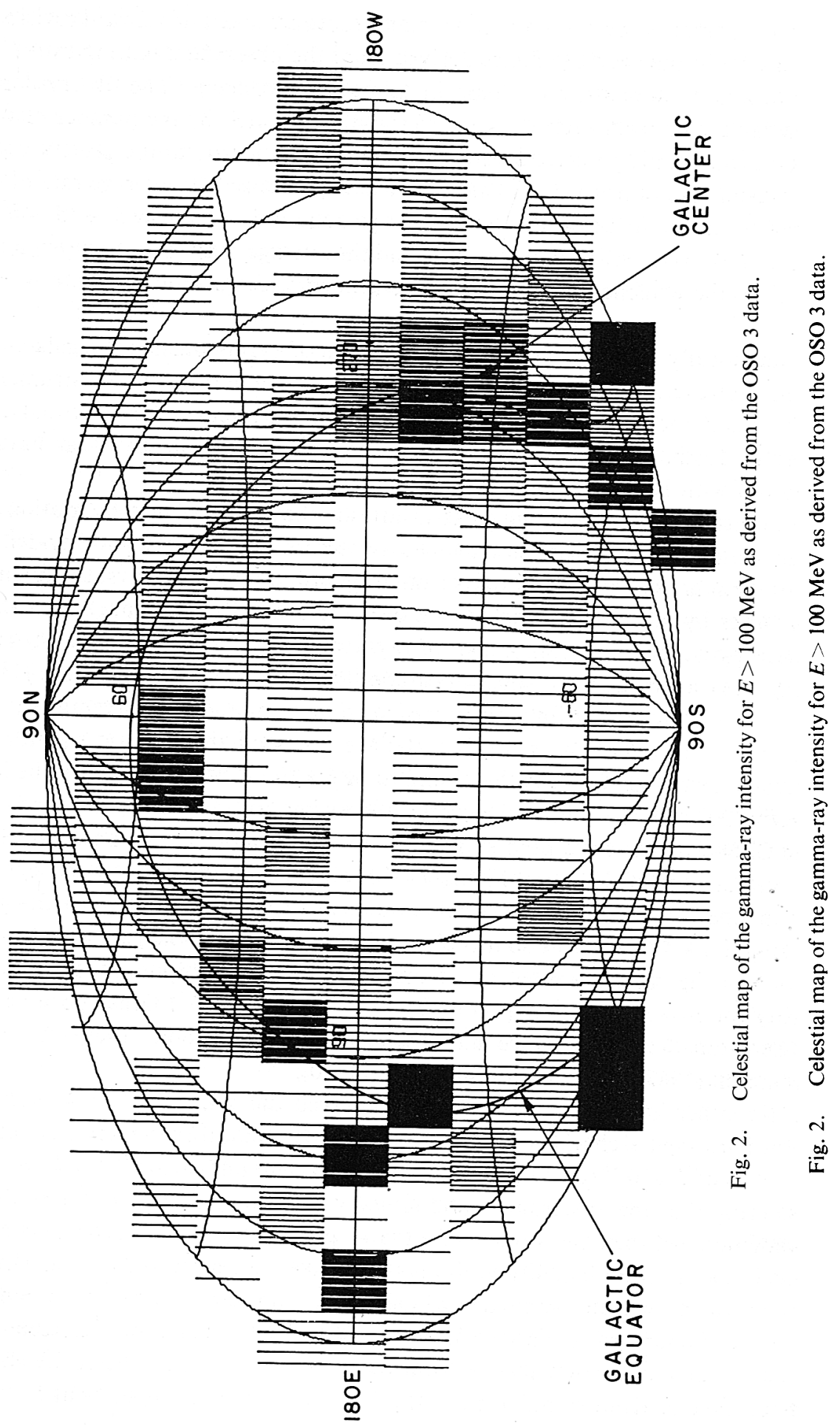


confidence level of $2.4 \times 10^{-5}\left(\mathrm{~cm}^{2} \mathrm{~s} \mathrm{rad}\right)^{-1}$ on the intensity above $50 \mathrm{MeV}$ from any source at the Frye location.

Evidence of a pulsating flux of $\sim 50 \mathrm{MeV}$ gamma rays from the Crab Nebula pulsar NP0532 has been reported by Leray et al. (1970) on the basis of data obtained in a balloon spark chamber experiment.

Various spark chamber observations have established upper limits in the neighborhood of $10^{-5}$ photons $\left(\mathrm{s} \mathrm{cm}^{2}\right)^{-1}$ at $E_{i}>10^{8} \mathrm{eV}$ on the fluxes of gamma rays from many potential discrete sources such as supernova remnants and radio galaxies.

The observation of galactic gamma rays has provided a new test of ideas about the distribution of matter and cosmic rays in the galaxy. Cavallo and Gould (1970) have re-examined the production of galactic gamma rays in the light of recent accelerator data on high energy nuclear interactions. Assuming that (1) the density of interstellar matter in the galaxy is that derived from $21 \mathrm{~cm}$ radio data, and (2) the intensity of cosmic rays in the galactic disk is the same as it is locally, they find a predicted equivalent line intensity of gamma rays from cosmic ray interactions which is in agreement with the revised OSO-3 results for the region away from the galactic center. On the other hand, the relatively high equivalent line intensity that is observed around the galactic center does not correspond to any substantial increase in the observed line density of atomic hydrogen. Thus either the cosmic ray intensity is much higher towards the center than it is locally, or else some process other than $\pi^{\circ}$ production must be operating.

\section{3. $10^{11}$ to $10^{13} \mathrm{eV}$}

In the energy range from $10^{11}$ to $10^{13} \mathrm{eV}$ the intensity of cosmic gamma rays is too low for detection by instruments of practical size which require the primary gamma-ray trajectory to intersect the instrument itself. However, a primary particle of such great energy generates an extensive shower of secondary particles when it enters the atmosphere. These secondary particles, in turn, emit optical Čerenkov radiation that can be observed by a ground-based detector which may lie at a considerable distance from the primary trajectory.

Air shower Cerenkov flashes observed from the ground appear in fast photographs as circular and elliptical blobs with angular dimensions on the order of one to several degrees. Although their shape and orientation depend on the orientation and distance of the air shower axis with respect to the observer, the center of intensity of any flash lies close to the direction from which the primary particle came. Thus Cerenkov flashes produced by air showers initiated by gamma rays from a discrete source will cluster about the direction of that source in a manner that should, in principle, make them distinguishable from the isotropically distributed flashes produced by ordinary showers initiated by charged particles. The technique is, of course, useful only in the search for discrete sources.

The most sensitive measurements reported to date are those obtained by Fazio et al. (1970) at mountain altitudes with a $10-\mathrm{m}$ steerable reflector. Table $\mathrm{I}$ is a list of upper limits on several of the most interesting potential discrete sources. Many others 
have also been examined by various investigators, all with negative results, and comprehensive lists can be found in the literature.

Although the results in this energy range are all negative so far, they have significant implications in certain cases. The upper limits on the gamma ray intensity from the Crab Nebula imply that the synchrotron electrons do not arise from high energy proton interactions which would also be a source of gamma rays, but that they must, instead, be directly accelerated (Cocconi, 1960). The high energy electrons $\left(\gtrsim 10^{12} \mathrm{eV}\right)$ which produce visible photons by synchrotron emission in the magnetic field also produce gamma-ray photons by inverse Compton scattering of these same synchrotron

TABLE I

Representative recent upper limits on the intensities of gamma rays from potential discrete sources

\begin{tabular}{lccc}
\hline Source & Ref. & $\begin{array}{l}\text { Energy threshold } \\
(\mathrm{eV})\end{array}$ & $\begin{array}{l}\text { Flux limit } \\
\text { photons }\left(\mathrm{s} \mathrm{cm}^{2}\right)^{-1}\end{array}$ \\
\hline Sag $\gamma$-1 & {$[1]$} & $5 \times 10^{i}$ & $2.4 \times 10^{-5}$ \\
Cygnus A & {$[2]$} & $1 \times 10^{8}$ & $\sim 1.0 \times 10^{-5}$ \\
Cygnus XR-1 & {$[2]$} & $1 \times 10^{8}$ & $\sim 1.0 \times 10^{-5}$ \\
Crab Nebula (steady) & {$[3]$} & $2.2 \times 10^{11}$ & $0.81 \times 10^{-10}$ \\
M-87 & & & \\
M-82 & {$[3]$} & $2.1 \times 10^{11}$ & $1.0 \times 10^{-10}$ \\
Pulsars & {$[3]$} & $3.2 \times 10^{11}$ & $0.71 \times 10^{-11}$ \\
NP0532 (Crab) & & & \\
NP0532 (Crab) & {$[3]$} & $2.2 \times 10^{11}$ & $6 \times 10^{-12}$ \\
CP1133 & {$[4]$} & $2 \times 10^{12}$ & $3 \times 10^{-11}$ \\
CP0950 & {$[4]$} & $1 \times 10^{12}$ & $1.0 \times 10^{-11}$ \\
& {$[4]$} & $1 \times 10^{12}$ & $1.7 \times 10^{-11}$
\end{tabular}

[1] Kniffen and Fichtel (1970).

[2] Valdez et al. (1969b).

[3] Fazio et al. (1970).

[4] Charman et al. (1969).

photons. Gould (1965) showed that a source which emits a given synchrotron flux must also emit a gamma-ray flux with an intensity proportional to $\mathrm{H}^{-(1+\alpha)}$ where $\alpha$ is the spectral index of the synchrotron spectrum. An upper limit on the gamma-ray flux therefore implies a lower limit on $\mathrm{H}$. The results of Fazio et al. (1970) place this lower limit at $3 \times 10^{-4} \mathrm{G}$.

The Crab Nebula has been examined for periodic emission from the pulsar NP0532 with negative results of $<6 \times 10^{-12}\left(\mathrm{~cm}^{2} \mathrm{~s}\right)^{-1}$ for $E_{\gamma}>2.2 \times 10^{11} \mathrm{eV}$ by Fazio et al. (1970) and $<3 \times 10^{-11}\left(\mathrm{~cm}^{2} \mathrm{~s}\right)^{-1}$ for $E_{\gamma}>10^{13} \mathrm{eV}$ by Charman et al. (1969). Comparable upper limits on several other pulsars have been reported (see Table I). 


\section{4. $10^{14}$ to $10^{16} \mathrm{eV}$}

An air shower generated by a primary particle with energy above $10^{14} \mathrm{eV}$ may reach the ground with enough of its structure intact to provide evidence as to whether its primary was a nucleus or a gamma ray. Specifically, the proportion of penetrating mu mesons in a shower initiated by a gamma ray should be far smaller than the average in showers initiated by nuclei. Such 'low mu' showers can be selectively detected with an air shower array which includes a heavily shielded penetrating particle detector in anticoincidence. In addition, one can determine the direction of the shower by timing the arrival of the shower front at various points in the detector array. Such a detector system is, in effect, a directional detector of gamma rays with energies greater than $10^{14} \mathrm{eV}$ which arrive from anywhere within the broad field of view defined by the atmospheric absorption of the air showers.

An experiment along these lines was conducted at $17000 \mathrm{ft}$ altitude by Toyoda et al. (1965). They demonstrated the existence of air showers with the low proportion

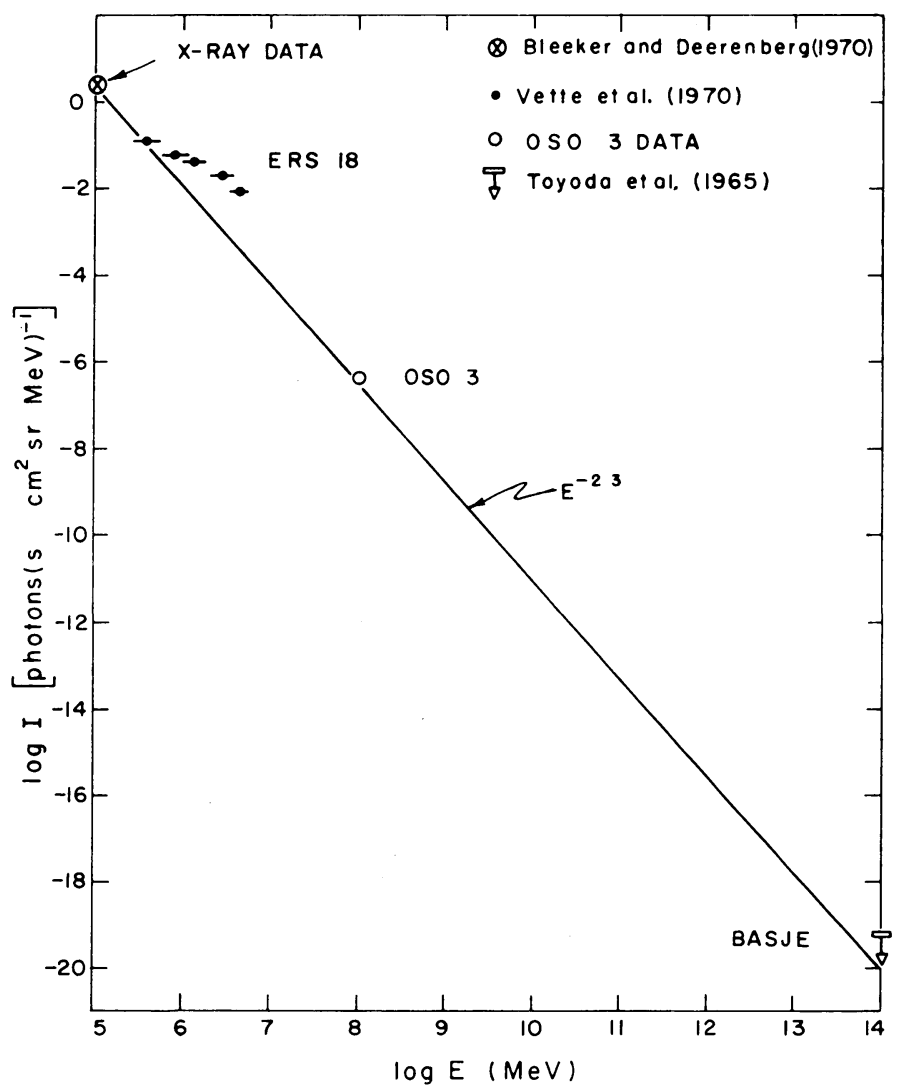

Fig. 3. Summary of the data on the cosmic diffuse gamma-ray spectrum from $\sim 100 \mathrm{keV}$ to $\sim 10^{14} \mathrm{eV}$. The ERS-18 points represent the amount by which the observed rates exceeded the rates calculated on the assumption that the spectrum is the $E^{-\cdots \cdot 3}$ power law shown. 
of mu mesons expected for gamma ray - induced showers. They mapped the arrival directions of over 600 of these events with an accuracy of $\pm 3^{\circ}$ and an effective energy threshold of $10^{14} \mathrm{eV}$. No statistically significant anisotropy was found. In the absence of such an isotropy there is no certain way to eliminate the possibility that most or all of such events are caused by primary protons which interact in a rare and peculiar way to transfer most of their energy to the electromagnetic component so that the resulting showers are as poor in mu mesons as would be expected for gamma-ray showers. Thus the experimental results could be used only to establish an upper limit on the isotropic gamma-ray flux which is less than $\leqslant 5 \times 10^{-4}$ times the flux of charged primaries above the same energy, or $5 \times 10^{-12}\left(\mathrm{~cm}^{2} \mathrm{~s} \mathrm{sr}\right)^{-1}$ for $E_{\gamma}>10^{14} \mathrm{eV}$.

Low-mu showers were also observed by Firkowski et al.(1962) at sea level. Although they were initially interpreted as being of photonic origin, new evidence was later cited to show that they were not (Catz et al., 1969).

The mean-free-paths of gamma rays with energies above the threshold required for pair production in collisions with starlight $\left(E \gtrsim 10^{12} \mathrm{eV}\right)$ and the universal $2.7^{\circ}$ radiation $\left(E \gtrsim 10^{14} \mathrm{eV}\right)$ are short compared to the Hubble distance. It is therefore likely that the spectrum of metagalactic gamma rays cuts off sharply above $10^{12} \mathrm{eV}$, and that it lies well below the upper limits set by the air shower experiments. On the other hand, the mean-free-path in the galaxy is comparable to the galactic diameter so that galactic sources above $10^{12} \mathrm{eV}$ may ultimately be observable.

\section{Summary}

Cosmic gamma rays have been observed in two regions of the spectrum - from 0.5 to $6 \mathrm{MeV}$ and from about $50 \mathrm{MeV}$ to several hundred MeV. Figure 3 is a summary of the available data on the isotropic and presumably metagalactic component. Above $50 \mathrm{MeV}$ a strong concentration of intensity is observed in a band of directions around the galactic equator. No definite evidence of a discrete source of gamma rays other than the galaxy itself has been observed at any energy.

\section{References}

Arnold, J. R., Metzger, A. E., Anderson, E. C. and VanDilla, M. A.: 1962, J. Geophys. Res. $67,4878$. Bleeker, J. A. M. and Deerenberg, A. J. M.: 1970, Astrophys. J. 159, 215.

Brecher, K. and Morrison, P.: 1969, Phys. Rev. Letters 23, 802.

Catz, Ph., Maze, R., Garvin, J., Przasnycki, M., Wdowczyk, J., and Zawadski, A.: 1969, Phys. Rev. Letters 23, 988.

Cavallo, G. and Gould, R. J.: 1970 (preprint).

Charman, W. N., Fruin, J. H., and Jelley, J. V.: 1969, Proc. Eleventh Intern. Conf. Cosmic Rays, Budapest.

Clark, G., Kraushaar, W., and Garmire, G.: 1968, Astrophys. J. Letters 153 L203.

Clayton, D. D. and Silk, J.: 1969, Astrophys. J. Letters 158, L43.

Critchfield, C. P., Ney, E. P., and Oleska, Sophie: 1952, Phys. Rev. 85, 461.

Fazio, G. G.: 1970, Nature 225, 905.

Fazio, G. G., Helmken, H. F., Rieke, G. H., and Weeks, T. C.: 1969, in L. Gratton (ed.) Non-Solar $\mathrm{X}$ - and Gamma-Ray Astronomy, IAU Symp. 37, 250.

Fazio, G. G., Weeks, T. C.. and Helmken, H. F.: 1970 (private communication). 
Feenberg, E. and Primakoff, H.: 1948, Phys. Rev. 73, 449.

Fichtel, C. and Kniffen, D.: 1968 (private communication).

Firkowski, R., Gawin, J., Zawadski, A., and Maze, R.: 1962, J. Phys. Soc. Japan 17 (Supl. AIII), 123.

Frye, G. M., Staib, J. A., Zych, A. P., Hopper, V. D., Rawlinson, W. R., and Thomas, J. A.: 1969, Nature 223, 1320.

Gould, G.: 1965, Phys. Rev. Letters 15, 577.

Hayakawa, S.: 1952, Prog. Theor. Phys. 8, 517.

Hulsizer, R. and Rossi, B.: 1949, Phy's. Rev. 76, 164.

Hutchinson, G. W.: 1962, Phil. Mag. 43, 847.

Hutchinson, G. W., Pearce, A. J., Ramsden, D., and Wills, R. D.: 1969, in L. Gratton (ed.) NonSolar X- and Gamma-Ray Astronomy, IAU Symp. 37, 300.

Kniffen, D. and Fichtel, C.: 1970, Astrophys. Letters 161, L157.

Leray, J. P., Parlier, B., Vasseur, J., Paul, J., Forichon, M., Agrinier, B., Buccheri, R., Scarsi, L., Boella, G., Maraschi, L., and Treves, A.: 1970 (paper given at COSPAR).

Perlow, G. J. and Kissinger, C. W.: 1951, Phys. Rev. 84, 572.

Sood, R. K.: 1969, Nature 222, 650.

Stecker, F. W.: 1969, Nature 224, 870.

Toyoda, Y., Suga, K., Murakami, K., Hasegawa, H., Shibata, S., Domingo, V., Escobar, I., Kamata, K., Bradt, H., Clark, G., and LaPointe, M.: 1965, Proc. Ninth Intern. Conf. Cosmic Rays, London 2, 708 .

Valdez, J. V. and Waddington, C. J.: 1969a, Astrophys. J. Letters 156, L85.

Valdez, J. V., Freier, P. S., and Waddington, C. J.: 1969b, Proceedings of the Eleventh Intern. Conf. Cosmic Rays, Budapest.

Vette, J. I., Gruber, G., Matteson, J. L., and Peterson, L.: 1970, Astrophys. J. Letters 160, L161.

\section{DISCUSSION}

R. Lüst: What is the energy range for the low and high energy events?

G. Clark: The response function of the OSO-3 detector has a threshold near $50 \mathrm{MeV}$ and rises to about $20 \%$ efficiency for forward incidence around $250 \mathrm{MeV}$. Thus the division between 'high' and 'low' energy events is roughly at $100 \mathrm{MeV}$.

K. Pinkau: Are the theoretical calculations by Cavallo absolute, or are they normalized to your results? I was interested to see the good agreement since the revision of your data by a factor of 3 did not seem to be sufficient to cover the original factor of $\sim 20$ that seemed to exist.

$G$. Clark: The calculation of the line intensity of galactic gamma rays by $\pi^{\circ}$ production by Cavallo and Gould assumes that the local cosmic ray intensity (corrected for solar modulation) pervades the disc, and that the column density of matter is given by the $21 \mathrm{~cm}$ survey data. With those two assumptions, the calculation is an absolute one so that the good agreement, with the OSO- 3 result (away from the galactic center) is significant evidence that the $\pi$ explanation is correct. 